Departamento de Matemática e Aplicações
Universidade do Minho
Caniversidade do Minho

\title{
On Paravector Valued Homogeneous Monogenic Polynomials with Binomial Expansion
}

\author{
H.R. Malonek ${ }^{a} \quad$ M.I. Falcão ${ }^{b}$
}

${ }^{a}$ Departamento de Matemática, Universidade de Aveiro, Portugal

${ }^{b}$ Departamento de Matemática e Aplicações, Universidade do Minho, Portugal

\section{Information}

Keywords:

Clifford Analysis, generalized Appell polynomials

Original publication:

Advances in Applied Clifford Analysis Volume 22, Issue 3 (2012), 789-801.

DOI: $10.1007 /$ s00006-012-0361-5

www.springer.com

\section{Introduction}

\subsection{Historical remarks}

During November 2005 Jaime Keller visited the University of Aveiro to give a course The Geometric Formulation of Physics from the view point of his START theory. Besides graduate and post-graduate physics and mathematics students, also members of our research group on Complex and Hypercomplex Analysis attended his lively and inspiring lectures. His dedicated teaching and long discussions after the lectures allowed the participants to get new and different insights into the historical developments of the World of Physics. The first author remembers very well Jaime's pride about his personal encounter as a young scientist with Paul Dirac and Eugene Wigner. Maybe this was one of the moments in his life which contributed to his neverresting enthusiasm for the foundation of this journal, which now is being published by Birkhäuser. Also, on the occasion of meetings in Mexico and Greece as well as during his last stay in Aveiro in May 2010 when 
Jaime delivered a talk on Matter and Space Time, the historical aspects of the interaction of Mathematics and Physics played a dominant role in our discussions.

For this reason, in this paper dedicated to the memory of Jaime Keller, we have chosen a problem connected with an old idea of R. Fueter. Fueter discussed it in detail in one of his first papers on analytic functions of a quaternionic variable in 1932 [12] after having announced his work on this subject already in 1928 at the IMU congress in Bologna [11]. In Section 3 of [12] entitled Zusammenhang mit den analytischen Funktionen einer komplexen Variablen (Connection with Analytic Functions of One Complex Variable), Fueter tried to relate his hypercomplex function theory to the theory of complex holomorphic functions by using them as generating functions (in general sense, not in the sense of formal power series) for regular quaternion-valued functions ( $\mathbb{H}$-holomorphic functions in [14]). Without explicitly mentioning the fact that an integer power of a quaternionic variable $z$ is not regular, he used basically the property of $w=\Delta z^{n}, n \in \mathbb{N}$, being a solution of $\Delta \Delta z^{n}=0$ (the title of his paper makes an indirect reference to this property). Since the square of the Laplace operator $\Delta \Delta$ can be factored by the generalized Cauchy-Riemann operator in $\mathbb{H}$, Fueter was successful in reaching his objective and in his approach to transforming a holomorphic function of a complex variable into one of a quaternionic variable (this is now called the Fueter transform [14]).

It was only about 60 years later when $\mathrm{H}$. Leutwiler, motivated mainly by his desire to overcome the problem of the non regular integer powers of a quaternionic variable $z$ without its implicit use in the form of $w=\Delta z^{n}$, created his "Modified Clifford Analysis" [18]. His leading idea was to change from the Euclidean metric to the hyperbolic metric because the power function is the conjugate gradient of a harmonic function defined with respect to the hyperbolic metric of the upper half plane.

Some years later, G. Laville and I. Ramadanoff in [17] solved the same problem, namely, the determination of a function class containing the power function, by another method. By doing so, they introduced a class of holomorphic Cliffordian functions. Instead of changing the metric, they changed the order of the differential equation to a higher one according to the number of real variables used. This way they succeeded in including the power functions in the kernel of their hypercomplex Cauchy-Riemann type operator of odd order.

A third way of overcoming the same problem while staying within the class of ordinary monogenic functions and without metric changes or increasing order, was realized by constructing polynomials with a behavior of power-like functions under hypercomplex differentiation in a series of articles by the authors of this paper $[10,23,6]$. Their ideas, based on Appell's concept of power-like polynomials [1], have allowed recently to develop a systematic study of Appell sequences as tools for other applied problems, like quasi-conformal mappings [21] or construction of classes of generalized classical polynomials [4, 5]. Since the construction of power-like monogenic functions was of general interest in Clifford analysis, the study of sets of Appell polynomials has developed meanwhile in several directions and has been realized with different methods and by different authors. We mention, for instance, [2] or [16]. Of particular interest remains to us the fact that in [16] a far reaching general representation theoretical method, based on Gelfand-Tsetlin bases, was developed.

Our aim in the present paper is to study Appell sequences from the view point of paravector valued homogeneous monogenic polynomials with binomial expansion. In this way we will show that we can, apart from trivial cases, distinguish between two different types of those polynomials with totally different structure.

\subsection{Basic notations}

As usual, let $\left\{e_{1}, e_{2}, \ldots, e_{n}\right\}$ be an orthonormal basis of the Euclidean vector space $\mathbb{R}^{n}$ with a non-commutative product according to the multiplication rules

$$
e_{k} e_{l}+e_{l} e_{k}=-2 \delta_{k l}, \quad k, l=1, \ldots, n,
$$

where $\delta_{k l}$ is the Kronecker symbol. The set $\left\{e_{A}: A \subseteq\{1, \ldots, n\}\right\}$ with

$$
e_{A}=e_{h_{1}} e_{h_{2}} \cdots e_{h_{r}}, \quad 1 \leq h_{1}<\cdots<h_{r} \leq n, \quad e_{\emptyset}=e_{0}=1,
$$

forms a basis of the $2^{n}$-dimensional Clifford algebra $C \ell_{0, n}$ over $\mathbb{R}$. Let $\mathbb{R}^{n+1}$ be embedded in $C \ell_{0, n}$ by identifying $\left(x_{0}, x_{1}, \ldots, x_{n}\right) \in \mathbb{R}^{n+1}$ with

$$
x=x_{0}+\underline{x} \in \mathcal{A}:=\operatorname{span}_{\mathbb{R}}\left\{1, e_{1}, \ldots, e_{n}\right\} \subset C \ell_{0, n} .
$$


Here, $x_{0}=\operatorname{Sc}(x)$ and $\underline{x}=\operatorname{Vec}(x)=e_{1} x_{1}+\cdots+e_{n} x_{n}$ are, the so-called, scalar and vector parts of the paravector $x \in \mathcal{A}$. The conjugate of $x$ is given by $\bar{x}=x_{0}-\underline{x}$ and its norm by $|x|=(x \bar{x})^{\frac{1}{2}}=\left(x_{0}^{2}+x_{1}^{2}+\right.$ $\left.\cdots+x_{n}^{2}\right)^{\frac{1}{2}}$.

To call attention to its relation to the complex Wirtinger derivatives, we use the following notation for a generalized Cauchy-Riemann operator in $\mathbb{R}^{n+1}, n \geq 1$ :

$$
\bar{\partial}:=\frac{1}{2}\left(\partial_{0}+\partial_{\underline{x}}\right), \quad \partial_{0}:=\frac{\partial}{\partial x_{0}}, \quad \partial_{\underline{x}}:=e_{1} \frac{\partial}{\partial x_{1}}+\cdots+e_{n} \frac{\partial}{\partial x_{n}} .
$$

$\mathcal{C}^{1}$-functions $f$ satisfying the equation $\bar{\partial} f=0$ (resp. $f \bar{\partial}=0$ ) are called left monogenic (resp. right monogenic). We suppose that $f$ is hypercomplex-differentiable in $\Omega$ in the sense of $[13,19]$, that is, it has a uniquely defined areolar derivative $f^{\prime}$ in each point of $\Omega$ (see also [20]). Then, $f$ is real-differentiable and $f^{\prime}$ can be expressed by real partial derivatives as $f^{\prime}=\partial f$ where, analogously to the generalized CauchyRiemann operator, we use $\partial:=\frac{1}{2}\left(\partial_{0}-\partial_{\underline{x}}\right)$ for the conjugate Cauchy-Riemann operator. Since a hypercomplex differentiable function belongs to the kernel of $\bar{\partial}$, it follows that, in fact, $f^{\prime}=\partial_{0} f=-\partial_{\underline{x}} f$ which is similar to the complex case.

In general, $C \ell_{0, n}$-valued functions defined in some open subset $\Omega \subset \mathbb{R}^{n+1}$ are of the form $f(z)=$ $\sum_{A} f_{A}(z) e_{A}$ with real valued $f_{A}(z)$. However, in several applied problems it is very useful to construct $\mathcal{A}$-valued monogenic functions as functions of a paravector with special properties, particularly if we study expansions of $C \ell_{0, n}$-valued functions in terms of special polynomial bases defined in $\mathbb{R}^{n+1}$. In this case we have

$$
f\left(x_{0}, \underline{x}\right)=\sum_{j=0}^{n} f_{j}\left(x_{0}, \underline{x}\right) e_{j}
$$

and left monogenic $(\bar{\partial} f=0)$ functions are also right monogenic functions $(f \bar{\partial}=0)$, a fact which follows easily by direct inspection of the corresponding real system of first order partial differential equations. In [24] such system was called a generalized Riesz system. In the case $n=2$, the system has the form

$$
\begin{aligned}
\frac{\partial}{\partial x_{0}} f_{0}-\frac{\partial}{\partial x_{1}} f_{1}-\frac{\partial}{\partial x_{2}} f_{2} & =0 \\
\frac{\partial}{\partial x_{0}} f_{1}+\frac{\partial}{\partial x_{1}} f_{0} & =0 \\
\frac{\partial}{\partial x_{0}} f_{2}+\frac{\partial}{\partial x_{2}} f_{0} & =0 \\
\frac{\partial}{\partial x_{1}} f_{2}-\frac{\partial}{\partial x_{2}} f_{1} & =0
\end{aligned}
$$

We use also the classical definition of sequences of Appell polynomials [1] adapted to the hypercomplex case.

Definition 1.1. A sequence of monogenic polynomials $\left(\mathcal{F}_{k}\right)_{k \geq 0}$ of exact degree $k$ is called a generalized Appell sequence with respect to $\partial$ if

1. $\mathcal{F}_{0}(x) \equiv 1$,

2. $\partial \mathcal{F}_{k}=k \mathcal{F}_{k-1}, k=1,2, \ldots$

Of course, the second condition is the essential one while the first condition is the usually applied normalization condition which can be changed to any constant different from zero.

\subsection{Some remarks on a special generalized Appell sequence in the context of Clifford Analysis}

The motivation for writing present paper came from some elementary but sometimes overlooked or superficially interpreted properties of already known homogeneous polynomials. Therefore, we finish our introduction with 
a subsection on the binomial expansion of a paravector valued homogeneous monogenic polynomial of degree $k$ and arbitrary $n$. This expansion was obtained in connection with the first construction of a generalized Appell sequence in the context of Clifford analysis in short notes by the authors some years ago $[9,10,22,23]$. The binomial expansion under consideration is

$$
\mathcal{P}_{k}^{n}(x)=\sum_{s=0}^{k}\left(\begin{array}{l}
k \\
s
\end{array}\right) c_{s}(n) x_{0}^{k-s} \underline{x}^{s}
$$

with $c_{0}(n) \equiv 1$ for arbitrary $n \geq 0$. A suitable choice of the coefficients $c_{s}(n)$ for $s \geq 1$ guarantees that the sequence $\mathcal{P}:=\left(\mathcal{P}_{k}^{n}\right)_{k \in \mathbb{N}}$ is an Appell sequence. For a general relationship between binomial expansions and Appell sequences we refer to [7].

Let us consider some interesting special cases of (1.2). In the general formula, the lower dimensional (and commutative) cases of a single real variable $x=x_{0}$, i.e., $n=0$, and a complex variable $x=x_{0}+e_{1} x_{1}$, i.e., $n=1$ with $e_{1}$ as imaginary unit, are both included and characterized by

$$
c_{s}(0)=c_{s}(1) \equiv 1, \quad s=1,2, \ldots
$$

However, since the real case means that $\underline{x} \equiv 0$, the sum in (1.2) contains in this case only the term $x_{0}^{k-0}=x_{0}^{k}$. In the complex case we have $\underline{x}=e_{1} x_{1}$ and (1.2) ranges from $c_{0}(1) x_{0}^{k}$ to $c_{k}(1) \underline{x}^{k}$ or, more concretely, the polynomials are given by

$$
\mathcal{P}_{k}^{1}(x)=\sum_{s=0}^{k}\left(\begin{array}{l}
k \\
s
\end{array}\right) x_{0}^{k-s} \underline{x}^{s}=\left(x_{0}+x_{1} e_{1}\right)^{k},
$$

which are, as it should be expected, the usual powers of the holomorphic variable $z=x_{0}+e_{1} x_{1}$.

We notice also a remarkable fact that for the first noncommutative case when $n=2$ and $\underline{x}=x_{1} e_{1}+x_{2} e_{2}$, the coefficients $c_{s}(2)$ are the generalized central binomial coefficients with weight $\frac{1}{2^{s}}$, i.e.,

$$
c_{s}(2)=\frac{1}{2^{s}}\left(\begin{array}{c}
s \\
\left\lfloor\frac{s}{2}\right\rfloor
\end{array}\right), \quad s=0,1, \ldots, k,
$$

where $\lfloor\cdot\rfloor$ is the floor function. In this connection it seems worthwhile to remember Catalan numbers which are the usual central binomial coefficients with $s=2 m$ but with weight $\frac{1}{m+1}$. Whereas in our case the weight divides the generalized central binomial coefficients by the sum of all binomial coefficients of degree $s$, the Catalan numbers weight divides by the number of all binomial coefficients of degree $m$.

The general formula for $c_{s}(n)$ when $n \geq 1$ and $s=1,2, \ldots$ can be written in a compact form as

$$
c_{s}(n)= \begin{cases}\frac{s ! !(n-2) ! !}{(n+s-1) ! !}, & \text { if } s \text { is odd; } \\ c_{s-1}(n), & \text { if } s \text { is even, }\end{cases}
$$

where, as usual, we define $(-1) ! !=0 ! !=1$.

In the case $n=3$ with $\underline{x}=x_{1} e_{1}+x_{2} e_{2}+x_{3} e_{3}$, corresponding to the quaternionic case, this formula reveals also some special values for $c_{s}(3)$, namely, the reciprocal values of all odd integers. That is, we have $c_{0}(3)=1$ and

$$
c_{s}(3)=\frac{1}{s+2}=c_{s+1}(3) \text { for } s=1,3,5, \ldots
$$

Two relations follow immediately from formula (1.2). They characterize the behavior of $\mathcal{P}_{k}^{n}(x)$ through its restriction to the real line $\underline{x}=0$ and to the hyperplane $x_{0}=0$.

Property 1.1. $\mathcal{P}_{k}^{n}\left(x_{0}\right)=\left.\mathcal{P}_{k}^{n}(x)\right|_{\underline{x}=0}=x_{0}^{k}$ for all $x_{0} \in \mathbb{R}, n \geq 0$.

Property 1.2. $\mathcal{P}_{k}^{n}(\underline{x})=\left.\mathcal{P}_{k}^{n}(x)\right|_{x_{0}=0}=c_{k}(n) \underline{x}^{k}, n \geq 1$.

These properties lead to a second binomial representation of $\mathcal{P}_{k}^{n}(x)$ in terms of its restrictions to the real line and the hyperplane $x_{0}=0$, namely,

$$
\mathcal{P}_{k}^{n}(x)=\sum_{s=0}^{k}\left(\begin{array}{l}
k \\
s
\end{array}\right) \mathcal{P}_{k-s}^{n}\left(x_{0}\right) \mathcal{P}_{s}^{n}(\underline{x}) .
$$


The first property guarantees that by restricting to $\underline{x}=0$ we obtain the classical and most simple real Appell sequence since $\mathcal{P}_{k}^{n}\left(x_{0}\right)=x_{0}^{k}$ for arbitrary $n \geq 0$. The other interpretation of this property is to say that the considered Appell sequence $\mathcal{P}:=\left(\mathcal{P}_{k}^{n}\right)_{k \in \mathbb{N}}$ is a hypercomplex continuation of the ordinary real Appell sequence $\mathcal{F}:=\left(x_{0}^{k}\right)_{k \in \mathbb{N}}$ into $\mathbb{R}^{n+1}, n \geq 1$.

For $n=1$, the second property characterizes the ordinary complex Appell sequence $\mathcal{P}_{k}^{1}(z)=z^{k}=$ $\left(x_{0}+x_{1} e_{1}\right)^{k}$ through its restriction to the values on the imaginary line by $\mathcal{P}_{k}^{1}\left(x_{1} e_{1}\right)=c_{k}(1) \underline{x}^{k}=\underline{x}^{k}$ as we should expect. We could also say that the complex Appell sequence $\mathcal{F}:=\left(z^{k}\right)_{k \in \mathbb{N}}$ is the CauchyKovalevskaya continuation from the imaginary line into the complex plane $\mathbb{C}$. Needless to say, this is dual to the usual situation where we consider the continuation from the real line to the complex plane.

The second property shows also clearly the difference in higher dimensional cases when $n \geq 2$ because in those cases the values of $c_{k}(n)$ are different from 1 for $k \geq 1$. From another point of view, it shows that $\mathcal{P}_{k}^{n}(x)$ is the Cauchy-Kovalevskaya continuation of $c_{k}(n) \underline{x}^{k}$ discussed in [3] but not of $\underline{x}^{k}$. This explains some difficulties in the justification of the hypercomplex continuations of holomorphic functions based formally on the integer powers of the vector part as in $\mathcal{P}_{k}^{n}(\underline{x})=\underline{x}^{k}$ and not really on $c_{k}(n) \underline{x}^{k}$ or the complex case with $\mathcal{P}_{k}^{1}(z)=z^{k}$ (see also [5]).

\section{The exclusive role of two types of homogeneous monogenic poly- nomials with binomial expansion}

The binomial expansion(1.5) contains $\mathcal{P}_{k}^{n}(\underline{x})=c_{k}(n) \underline{x}^{k}, n \geq 0$, as a natural generalization of the integer power of the imaginary part $\left(x_{1} e_{1}\right)^{k}$ compared with (1.3). The coefficients $c_{k}(n) \neq 0$ for $n \geq 1$ obviously reflect the deviation from the complex case in correspondence to the number $n$ of components in the vector part $\underline{x}$. But why should the restriction to the hyperplane $x_{0}=0$ of the polynomials $\mathcal{P}_{k}^{n}(x)$ of an Appell sequence only be of the form expressed in Property 1.2?

To answer this question and to obtain a better understanding of all possible paravector valued homogeneous monogenic polynomials that can be used for the construction of sequences of generalized Appell polynomials in the context of Clifford Analysis, we admit now a more general form of the vector part of the first degree polynomial in the Appell sequence. This means that instead of $\underline{x}=\sum_{j=1}^{n} x_{j} e_{j}$ we choose now $n$ linear functions $X_{j}=X_{j}\left(x_{1}, \ldots, x_{n}\right)$ as part of $\underline{X}=\sum_{j=1}^{n} X_{j}\left(x_{1}, \ldots, x_{n}\right) e_{j}$ and look for the conditions that

$$
\mathcal{P}_{k}^{n}\left(x_{0}, \hat{x}\right)=\sum_{s=0}^{k}\left(\begin{array}{l}
k \\
s
\end{array}\right) d_{s}(n) x_{0}^{k-s} \underline{X}^{s}=\sum_{s=0}^{k}\left(\begin{array}{l}
k \\
s
\end{array}\right) \mathcal{P}_{k-s}^{n}\left(x_{0}\right) \mathcal{P}_{s}^{n}(\underline{X}),
$$

with some suitably chosen real coefficients $d_{s}(n)$, form a sequence of Appell polynomials. The preserved separation of the variable $x_{0}$ is again motivated by the intention of keeping the ordinary real Appell sequence as the restriction on the real line where $\underline{X} \equiv 0$ as a consequence of being a degree one homogeneous (linear) polynomial.

It is trivial to recognize that these polynomials are really paravector valued homogeneous monogenic polynomials of degree $k$ since all even integer powers of the pure vector valued first degree homogeneous function $\underline{X}$ are real valued and, as a consequence of this, all odd powers of $\underline{X}$ are pure vectors. For normalizing reasons we suppose also that $d_{0}(n) \equiv 1, n \geq 0$. It is also evident how the $\mathcal{P}_{k}\left(x_{0}, \hat{x}\right)$ maintain in an analogous form Properties 1.1 and 1.2 of $\mathcal{P}_{k}^{n}(x)$ as described in the last subsection.

We study now in detail the case of paravector valued polynomials of three real variables with binomial expansion of the form (2.1). More precisely, we consider homogeneous monogenic polynomials $\mathcal{P}_{k}^{2}\left(x_{0}, \hat{x}\right):=$ $\mathcal{P}_{k}\left(x_{0}, \hat{x}\right)$ with the binomial expansion of the form

$$
\mathcal{P}_{k}\left(x_{0}, \hat{x}\right)=\sum_{s=0}^{k}\left(\begin{array}{l}
k \\
s
\end{array}\right) d_{s} x_{0}^{k-s}\left(X_{1}\left(x_{1}, x_{2}\right) e_{1}+X_{2}\left(x_{1}, x_{2}\right) e_{2}\right)^{s},
$$

where $X_{j}=X_{j}\left(x_{1}, x_{2}\right), j=1,2$, are two real valued linear functions and $d_{0}=1$.

Theorem 2.1. For polynomials of the form (2.2), we consider the following two different situations: 
Case $1 \partial_{1} X_{1} \cdot \partial_{2} X_{2}=0$

This case corresponds to the trivial Appell polynomials,

$$
\mathcal{P}_{k}\left(x_{0}, x_{2}\right)=\sum_{s=0}^{k}\left(\begin{array}{l}
k \\
s
\end{array}\right) x_{0}^{k-s}\left(x_{2} e_{2}\right)^{s}=\left(x_{0}+x_{2} e_{2}\right)^{k}
$$

and

$$
\mathcal{P}_{k}\left(x_{0}, x_{1}\right)=\sum_{s=0}^{k}\left(\begin{array}{l}
k \\
s
\end{array}\right) x_{0}^{k-s}\left(x_{1} e_{1}\right)^{s}=\left(x_{0}+x_{1} e_{1}\right)^{k}
$$

associated to $\partial_{1} X_{1}=0$ and $\partial_{2} X_{2}=0$, respectively.

Case $2 \partial_{1} X_{1} \cdot \partial_{2} X_{2} \neq 0$

In this case, there are exactly two different types of non-trivial Appell polynomials, namely

1. The Appell polynomials (1.2),

$$
\mathcal{P}_{k}\left(x_{0}, \hat{x}\right)=\mathcal{P}_{k}^{2}\left(x_{0}, \underline{x}\right)=\sum_{s=0}^{k}\left(\begin{array}{l}
k \\
s
\end{array}\right) c_{s}(2) x_{0}^{k-s}\left(x_{1} e_{1}+x_{2} e_{2}\right)^{s},
$$

corresponding to the case $\partial_{1} X_{2}=\partial_{2} X_{1}=0$.

2. The polynomials isomorphic to the complex integer powers of $z$,

$$
\begin{aligned}
\mathcal{P}_{k}\left(x_{0}, \hat{x}\right) & =\sum_{s=0}^{k}\left(\begin{array}{l}
k \\
s
\end{array}\right) x_{0}^{k-s}\left(\left(i_{1} x_{1}+i_{2} x_{2}\right)\left(i_{1} e_{1}+i_{2} e_{2}\right)\right)^{s} \\
& =\left(x_{0}+\left(i_{1} x_{1}+i_{2} x_{2}\right)\left(i_{1} e_{1}+i_{2} e_{2}\right)\right)^{k},
\end{aligned}
$$

with a real unit parameter-vector $\left(i_{1}, i_{2}\right)$, i.e., with $i_{1}^{2}+i_{2}^{2}=1$, when $\partial_{1} X_{2}=\partial_{2} X_{1} \neq 0$.

Proof. The first degree polynomial in the sequence (2.2) has the form

$$
\mathcal{P}_{1}\left(x_{0}, \hat{x}\right)=\mathcal{P}_{1}\left(x_{0}\right)+\mathcal{P}_{1}(\underline{X})=x_{0}+d_{1}\left(X_{1}\left(x_{1}, x_{2}\right) e_{1}+X_{2}\left(x_{1}, x_{2}\right) e_{2}\right) .
$$

Since its vector components are first degree homogeneous functions, it is clear that they can be written explicitly in the form

$$
X_{j}\left(x_{1}, x_{2}\right)=a_{j 1} x_{1}+a_{j 2} x_{2}, \quad j=1,2,
$$

with some real $a_{j m}, j, m=1,2$. Moreover, as a consequence of the last equation in the Riesz system (1.1) (the compatibility condition $\partial_{1} X_{2}=\partial_{2} X_{1}$ ), functions $X_{j}$ are in fact of the form

$$
X_{1}\left(x_{1}, x_{2}\right)=a_{11} x_{1}+\lambda x_{2} \quad \text { and } \quad X_{2}\left(x_{1}, x_{2}\right)=\lambda x_{1}+a_{22} x_{2} \text {, }
$$

for some real $\lambda$. Therefore,

$$
\mathcal{P}_{1}\left(x_{0}, \hat{x}\right)=x_{0}+d_{1}\left(\left(a_{11} x_{1}+\lambda x_{2}\right) e_{1}+\left(\lambda x_{1}+a_{22} x_{2}\right) e_{2}\right)
$$

is a monogenic polynomial assuming

$$
\bar{\partial} \mathcal{P}_{1}\left(x_{0}, \hat{x}\right)=\frac{1}{2}\left(1-d_{1}\left(a_{11}+a_{22}\right)\right)=0 .
$$

Last relation holds provided

$$
a_{11}+a_{22} \neq 0
$$

and

$$
d_{1}=\frac{1}{a_{11}+a_{22}} .
$$


Now we consider

$$
\begin{aligned}
\mathcal{P}_{2}\left(x_{0}, \hat{x}\right)=x_{0}^{2}+2 x_{0} d_{1}\left(\left(a_{11} x_{1}+\lambda x_{2}\right) e_{1}+\left(\lambda x_{1}+a_{22} x_{2}\right) e_{2}\right) & \\
& +d_{2}\left(\left(a_{11} x_{1}+\lambda x_{2}\right) e_{1}+\left(\lambda x_{1}+a_{22} x_{2}\right) e_{2}\right)^{2}
\end{aligned}
$$

and check under what condition $\bar{\partial} \mathcal{P}_{2}\left(x_{0}, \hat{x}\right)=0$. By a straightforward computation we obtain

$$
\begin{aligned}
\bar{\partial} \mathcal{P}_{2}\left(x_{0}, \hat{x}\right)=\left(\left(d_{1} a_{11}-d_{2} a_{11}^{2}-d_{2} \lambda^{2}\right) x_{1}\right. & \left.+\left(d_{1}-d_{2} a_{11}-d_{2} a_{22}\right) \lambda x_{2}\right) e_{1} \\
& +\left(\left(d_{1}-d_{2} a_{11}-d_{2} a_{22}\right) \lambda x_{1}+\left(d_{1} a_{22}-d_{2} a_{22}^{2}-d_{2} \lambda^{2}\right) x_{2}\right) e_{2} .
\end{aligned}
$$

The right choice of the coefficient $d_{2}$ must now guarantee that $\mathcal{P}_{2}\left(x_{0}, \hat{x}\right)$ is monogenic. Using $(2.10)$, this problem is equivalent to the solution of the system

$$
\begin{aligned}
& \left(\frac{a_{11}}{a_{11}+a_{22}}-d_{2}\left(a_{11}^{2}+\lambda^{2}\right)\right) x_{1}+\left(\frac{1}{a_{11}+a_{22}}-d_{2}\left(a_{11}+a_{22}\right)\right) \lambda x_{2}=0, \\
& \left(\frac{1}{a_{11}+a_{22}}-d_{2}\left(a_{11}+a_{22}\right)\right) \lambda x_{1}+\left(\frac{a_{22}}{a_{11}+a_{22}}-d_{2}\left(a_{22}^{2}+\lambda^{2}\right)\right) x_{2}=0,
\end{aligned}
$$

which in turn is equivalent, for independent $x_{1}, x_{2}$, to the following system

$$
\begin{aligned}
\frac{a_{11}}{a_{11}+a_{22}}-d_{2}\left(a_{11}^{2}+\lambda^{2}\right) & =0, \\
\left(\frac{1}{a_{11}+a_{22}}-d_{2}\left(a_{11}+a_{22}\right)\right) \lambda & =0, \\
\frac{a_{22}}{a_{11}+a_{22}}-d_{2}\left(a_{22}^{2}+\lambda^{2}\right) & =0 .
\end{aligned}
$$

We consider now several different situations:

(i) When $a_{11}=0$, we get from (2.9) that $a_{22} \neq 0$ and, therefore,

$$
\lambda=0 \quad \text { and } \quad d_{2}=\frac{1}{a_{22}^{2}}=d_{1}^{2} .
$$

The final form of the monogenic polynomials $\mathcal{P}_{1}\left(x_{0}, \hat{x}\right)$ in (2.8) and $\mathcal{P}_{2}\left(x_{0}, \hat{x}\right)$ in $(2.11)$ are as follows

$$
\begin{aligned}
& \mathcal{P}_{1}\left(x_{0}, \hat{x}\right)=x_{0}+x_{2} e_{2}, \\
& \mathcal{P}_{2}\left(x_{0}, \hat{x}\right)=x_{0}^{2}+2 x_{0} x_{2} e_{2}-x_{2}^{2}=\left(x_{0}+x_{2} e_{2}\right)^{2} .
\end{aligned}
$$

Repeating again the same process, we get $d_{k}=\frac{1}{a_{22}^{k}}, k=1,2 \ldots$, with the effect that

$$
\mathcal{P}_{k}\left(x_{0}, \hat{x}\right)=\left(\mathcal{P}_{1}\left(x_{0}, \hat{x}\right)\right)^{k},
$$

which can be confirmed by the particular cases (2.13). Relation (2.3) is proved since $a_{11}=\partial_{1} X_{1}$, $a_{22}=\partial_{2} X_{2}$, and $\lambda=\partial_{1} X_{2}=\partial_{2} X_{1}$.

(ii) The case $a_{22}=0$, leading to (2.4), is obviously of the same nature and we skip the proof. Both cases lead to Appell sequences isomorphic to the complex integer power sequence $z^{k}$.

Of course, the result is evident and it could have been guessed directly if only we had recalled that the generalized Cauchy-Riemann operator $\bar{\partial}$ acts on functions defined in the hyperplane $x_{1}=0$ like the complex Wirtinger operator $\partial_{\bar{z}}=\frac{1}{2}\left(\partial_{0}+e_{2} \partial_{2}\right)$ and the hypercomplex derivative reduces to the corresponding complex derivative.

(iii) If $a_{11} a_{22} \neq 0$ and $\lambda=0$ then we have from (2.12)

$$
\frac{1}{a_{11}+a_{22}}=d_{2} a_{11} \text { and } \frac{1}{a_{11}+a_{22}}=d_{2} a_{22}
$$

and, therefore, $a_{11}=a_{22}=a$. The consequence for $\mathcal{P}_{1}\left(x_{0}, \hat{x}\right)$ in view of $(2.8)$ is

$$
\begin{aligned}
\mathcal{P}_{1}\left(x_{0}, \hat{x}\right) & =x_{0}+\frac{1}{2 a}\left(a x_{1} e_{1}+a x_{2} e_{2}\right) \\
& =x_{0}+\frac{1}{2}\left(x_{1} e_{1}+x_{2} e_{2}\right)=\mathcal{P}_{1}\left(x_{0}, \underline{x}\right) .
\end{aligned}
$$

As we can see, this case corresponds to the well studied sequence of Appell polynomials (1.2) and this proves relation $(2.5)$. 
(iv) If $a_{11} a_{22} \neq 0$ and $\lambda \neq 0$, we obtain from (2.12) the following system:

$$
\begin{aligned}
\frac{a_{11}}{a_{11}+a_{22}}-d_{2}\left(a_{11}^{2}+\lambda^{2}\right) & =0, \\
\frac{1}{a_{11}+a_{22}}-d_{2}\left(a_{11}+a_{22}\right) & =0, \\
\frac{a_{22}}{a_{11}+a_{22}}-d_{2}\left(a_{22}^{2}+\lambda^{2}\right) & =0,
\end{aligned}
$$

and the second equation of (2.14) yields $d_{2}=\frac{1}{\left(a_{11}+a_{22}\right)^{2}}$. In addition, this value of $d_{2}$ helps now to establish an important relationship between $\lambda$ and the other coefficients, namely,

$$
\begin{aligned}
& a_{11}\left(a_{11}+a_{22}\right)-\left(a_{11}^{2}+\lambda^{2}\right)=0 \\
& a_{22}\left(a_{11}+a_{22}\right)-\left(a_{22}^{2}+\lambda^{2}\right)=0 .
\end{aligned}
$$

This means in both cases that the product of $a_{11}$ and $a_{22}$ must be positive since (2.15) simplifies to

$$
a_{11} a_{22}=\lambda^{2} .
$$

Some elementary observations are now needed for the last step of the proof. Since a positive product of two real numbers is only possible, if both are of the same sign, it is true that all three numbers $a_{11}, a_{22}$, and $a_{11}+a_{22}$ are of the same sign. Therefore it is also true that their quotients $\frac{a_{11}}{a_{11}+a_{22}}$ and $\frac{a_{22}}{a_{11}+a_{22}}$ are positive and can be written as squares of their roots $i_{1}$ and $i_{2}$ in the form

$$
i_{1}^{2}:=\frac{a_{11}}{a_{11}+a_{22}} \quad \text { and } \quad i_{2}^{2}:=\frac{a_{22}}{a_{11}+a_{22}} .
$$

Using both expressions together with (2.15), we recognize that

$$
i_{1}^{2} i_{2}^{2}=\frac{a_{11} a_{22}}{\left(a_{11}+a_{22}\right)^{2}}=\frac{\lambda^{2}}{\left(a_{11}+a_{22}\right)^{2}} .
$$

In the last step of the proof we apply now the aforementioned relations for substituting the coefficients in $\mathcal{P}_{1}\left(x_{0}, \hat{x}\right)$ by a chosen pair of those roots $i_{1}$ and $i_{2}$ as defined in (2.17). Thus, we have from (2.8):

$$
\begin{aligned}
\mathcal{P}_{1} & \left(x_{0}, \hat{x}\right) \\
& =x_{0}+\left(\frac{a_{11}}{a_{11}+a_{22}} x_{1}+\frac{\lambda}{a_{11}+a_{22}} x_{2}\right) e_{1}+\left(\frac{\lambda}{a_{11}+a_{22}} x_{1}+\frac{a_{22}}{a_{11}+a_{22}} x_{2}\right) e_{2} \\
& =x_{0}+\left(i_{1}^{2} x_{1}+i_{1} i_{2} x_{2}\right) e_{1}+\left(i_{1} i_{2} x_{1}+i_{2}^{2} x_{2}\right) e_{2} \\
& =x_{0}+\left(i_{1} x_{1}+i_{2} x_{2}\right) i_{1} e_{1}+\left(i_{1} x_{1}+i_{2} x_{2}\right) i_{2} e_{2} \\
& =x_{0}+\left(i_{1} x_{1}+i_{2} x_{2}\right)\left(i_{1} e_{1}+i_{2} e_{2}\right) .
\end{aligned}
$$

Notice that from (2.17) we obtain $i_{1}^{2}+i_{2}^{2}=1$. In this case it follows immediately that

$$
\left(i_{1} e_{1}+i_{2} e_{2}\right)^{2}=-\left(i_{1}^{2}+i_{2}^{2}\right)=-1 \text {. }
$$

The latter shows the isomorphism of the structure of $\mathcal{P}_{k}\left(x_{0}, \hat{x}\right)$ with $z^{k}$ and it implies also that $\mathcal{P}_{k}\left(x_{0}, \hat{x}\right)=\left(\mathcal{P}_{1}\left(x_{0}, \hat{x}\right)\right)^{k}$.

\section{Concluding remarks}

First of all, we would like to mention that Case 2.2 as well as Case 2.1 of the well studied Appell polynomials $\mathcal{P}_{k}^{n}\left(x_{0}, \underline{x}\right)$ admits a direct generalization to arbitrary dimension if, instead of $\left(i_{1}, i_{2}\right)$, a general unit parameter vector $\left(i_{1}, \ldots, i_{n}\right)$ is used and so (2.19) generalizes to

$$
\left(i_{1} e_{1}+\cdots+i_{n} e_{n}\right)^{2}=-\left(i_{1}^{2}+\cdots i_{n}^{2}\right)=-1 .
$$

The proof that these polynomials and $\mathcal{P}_{k}^{n}\left(x_{0}, \underline{x}\right)$ are the only possible non trivial extension of $z^{k}$ can follow the line described in the case of $n=2$. The requirement of being monogenic and, at the same time, satisfying 
the Appell sequence property was here the key step. This approach also works for $n>2$ but then it needs more advanced algebraic tools.

The possibility to generate monogenic functions from holomorphic functions by using generalized powers

$$
\mathcal{P}_{k}\left(x_{0}, \hat{x}\right)=\left(x_{0}+\left(i_{1} x_{1}+i_{2} x_{2}\right)\left(i_{1} e_{1}+i_{2} e_{2}\right)\right)^{k}
$$

was first mentioned in [8]. It was extended later by using several hypercomplex variables in [6]. The result presented here confirms our feeling about the exclusive role of both approaches. Moreover, Case 2.2 seems to be more flexible than Case 2.1 which only gives axially symmetric polynomials. Results of ongoing research in applications of monogenic Appell sequences confirm this fact.

The paper [15] discussed the construction of $\mathcal{P}_{k}^{n}\left(x_{0}, \underline{x}\right)$ but only for an odd integer $n$ by the so-called Fueter-Sce mapping where an essential ingredient (already considered by Fueter in [12]) was the use of a variable imaginary unit given by $\omega(x)=\frac{x}{|x|}$. Case 2.2 shows that also (and independently from the dimension) the constant imaginary vector $\hat{\imath}=\left(i_{1} e_{1}+\cdots+i_{n} e_{n}\right)$ serves very well in the construction of an Appell sequence.

Whereas the behavior of $\mathcal{P}_{k}^{n}\left(x_{0}, \underline{x}\right)$ is that of a non commutative construction expressed by $\mathcal{P}_{k}^{n}\left(x_{0}, \underline{x}\right) \neq$ $\left(\mathcal{P}_{1}^{n}\left(x_{0}, \underline{x}\right)\right)^{k}$, we see that the property $\mathcal{P}_{k}\left(x_{0}, \hat{x}\right)=\left(\mathcal{P}_{1}\left(x_{0}, \hat{x}\right)\right)^{k}$ reveals the commutative character of Case 2.2 .

If one uses, instead of the classical Appell sequence property $\mathcal{F}_{0}(x) \equiv 1$ from Definition 1.1 , a generalized constant monogenic polynomial as an initial value like it was done in [2], then the set of $\mathcal{P}_{k}^{n}\left(x_{0}, \underline{x}\right)$ can also be extended and used in several applications, for instance in elasticity problems.

\section{References}

[1] P. Appell, Sur une classe de polynômes. Ann. Sci. École Norm. Sup. 9 (1880), no. 2, 119-144.

[2] S. Bock and K. Gürlebeck, On a Generalized Appell System and Monogenic Power Series. Math. Methods Appl. Sci. 33 (2010), no. 4, 394-411.

[3] F. Brackx, R. Delanghe, and F. Sommen, Clifford Analysis. Pitman, Boston-London-Melbourne, 1982.

[4] I. Cação, M. I. Falcão, and H. R. Malonek, Laguerre Derivative and Monogenic Laguerre Polynomials: An Operational Approach. Math. Comput. Model. 53 (2011), no. 5-6, 1084-1094.

[5] I. Cação, M. I. Falcão, and H. R. Malonek, On Generalized Hypercomplex Laguerre-Type Exponentials and Applications. In Computational Science and Its Applications - ICCSA 2011, B. Murgante, O. Gervasi, A. Iglesias, D. Taniar, and B. Apduhan, Eds., Lecture Notes in Computer Science, vol. 6784, SpringerVerlag, Berlin, Heidelberg, 2011, 271-286.

[6] I. Cação and H. R. Malonek, On Complete Sets of Hypercomplex Appell Polynomials. In AIP Conference Proceedings, Th. E. Simos, G. Psihoyios, and Ch. Tsitouras, Eds., vol. 1048, 2008, 647-650.

[7] B. C. Carlson, Polynomials Satisfying a Binomial Theorem. J. Math. Anal. Appl. 32 (1970), 543-558.

[8] J. Cruz, M. I. Falcão, and H. R. Malonek, 3D-Mappings and Their Approximations by Series of Powers of a Small Parameter. In 17th Inter. Conf. on the Appl. of Computer Science and Mathematics on Architecture and Civil Engineering, Weimar, 2006.

[9] M. I. Falcão, J. Cruz, and H. R. Malonek, Remarks on the Generation of Monogenic Functions. In 17th Inter. Conf. on the Appl. of Computer Science and Mathematics on Architecture and Civil Engineering, Weimar, 2006.

[10] M. I. Falcão and H. R. Malonek, Generalized Exponentials Through Appell Sets in $\mathbb{R}^{n+1}$ and Bessel Functions. In AIP Conference Proceedings, Th. E. Simos, G. Psihoyios, and Ch. Tsitouras, Eds., vol. 936, 2007, 738-741.

[11] R. Fueter, Über Funktionen einer Quaternionenvariablen., Atti Congresso Bologna 2, 145, 1930.

[12] R. Fueter, Analytische Funktionen einer Quaternionenvariablen, Comment. Math. Helv. 4 (1932), 9-20. 
[13] K. Gürlebeck and H. R. Malonek, A Hypercomplex Derivative of Monogenic Functions in $\mathbb{R}^{n+1}$ and Its Applications. Complex Variables Theory Appl. 39 (1999), 199-228.

[14] K. Gürlebeck, K. Habetha, and W. Sprößig, Holomorphic Functions in the Plane and n-Dimensional Space. Birkhäuser Verlag, Basel, 2008, Translated from the 2006 German original.

[15] N. Gürlebeck, On Appell Sets and the Fueter-Sce Mapping. Adv. Appl. Clifford Algebras 19 (2009), no. 1, 51-61.

[16] R. Lávička, Canonical Bases for sl(2,c)-Modules of Spherical Monogenics in Dimension 3. Archivum Mathematicum 46 (2010), 339-349.

[17] G. Laville and I. Ramadanoff, Holomorphic Cliffordian Functions. Adv. Appl. Clifford Algebras 8 (1998), 323-340.

[18] H. Leutwiler, Modified Quaternionic Analysis in $\mathbb{R}^{3}$. Complex Variables, Theory Appl. 20 (1992), 19-51.

[19] H. R. Malonek, A New Hypercomplex Structure of the Euclidean Space $\mathbb{R}^{m+1}$ and the Concept of Hypercomplex Differentiability. Complex Variables 14 (1990), 25-33.

[20] H. R. Malonek, Selected Topics in Hypercomplex Function Theory. In Clifford Algebras and Potential Theory, S.-L. Eriksson, Ed., 7, University of Joensuu, 2004, 111-150.

[21] H. R. Malonek and R. De Almeida, A Note on a Generalized Joukowski Transformation. Appl. Math. Lett. 23 (2010), no. 10, 1174-1178.

[22] H. R. Malonek and M. I. Falcão, 3D-Mappings Using Monogenic Functions. Wiley-VCH, Weinheim, 2006, 615-619.

[23] H. R. Malonek and M. I. Falcão, Special Monogenic Polynomials-Properties and Applications. In AIP Conference Proceedings, Th. E. Simos, G. Psihoyios, and Ch. Tsitouras, Eds., vol. 936, 2007, 764-767.

[24] E. M. Stein and G. Weiss, Generalization of the Cauchy-Riemann Equations and Representations of the Rotation Group. Amer. J. Math. 90 (1968), 163-196. 